\title{
Propofol inhibits Wnt signaling and exerts anticancer activity in glioma cells
}

\author{
WEI XU ${ }^{1 *}$, JIWEI ZHENG ${ }^{1 *}$, SHIJIE BIE $^{1}$, LIUYU KANG ${ }^{1}$, \\ QINGJUN MAO ${ }^{1}$, WEIWEI LIU ${ }^{1}$, JINXIN GUO ${ }^{1}$, JUAN LU ${ }^{2}$ and RUI XIA ${ }^{1}$ \\ ${ }^{1}$ Department of Anesthesiology; ${ }^{2}$ Operating Room, The First People's Hospital of Jingzhou, \\ The First Affiliated Hospital of Yangtze University, Jingzhou, Hubei 434000, P.R. China
}

Received May 22, 2017; Accepted December 11, 2017

DOI: $10.3892 / \mathrm{ol} .2018 .8606$

\begin{abstract}
Aberrant activation of Wnt signaling is implicated in gliomagenesis. Propofol, the most commonly used intravenous anesthetic agent in clinics, exhibits potent antitumor activity in a variety of cancer cells through different mechanisms. However, the role of propofol on Wnt signaling and glioma cell growth remains to be fully elucidated. In the present study, propofol was identified as a potent inhibitor of Wnt signaling. In $293 \mathrm{~T}$ cells transfected with Wnt 1 or Wnt3 expression plasmids or treated with Wnt3A-conditioned medium, propofol significantly inhibited the transcriptional activity of the SuperTopFlash reporter and the expression of Wnt target genes. The inhibitory effect of propofol on Wnt signaling was also observed in glioma cells. Further experiments demonstrated that propofol suppressed glioma cell growth by decreasing cell proliferation and enhancing cell apoptosis. Finally, the potential antitumor efficiency of propofol was confirmed using xenograft experiments in vivo. Taken together, the results indicated a novel mechanism for the anticancer activity of propofol and provide supporting evidence for its use as a prospective anticancer drug to treat glioma in patients with deregulated Wnt signaling.
\end{abstract}

Correspondence to: Dr Rui Xia, Department of Anesthesiology, The First People's Hospital of Jingzhou, The First Affiliated Hospital of Yangtze University, 8 Hangkong Road, Shashi, Jingzhou, Hubei 434000, P.R. China

E-mail: xiaruijzyy@126.com

Dr Juan Lu, Operating Room, The First People's Hospital of Jingzhou, The First Affiliated Hospital of Yangtze University, 8 Hangkong Road, Shashi, Jingzhou, Hubei 434000, P.R. China

E-mail: lujunasss@yeah.net

\section{*Contributed equally}

Abbreviations: APC, adenomatous polyposis coli; CK1, casein kinase 1; GSK3 $\beta$, glycogen synthase kinase $3 \beta$; LRP5, lipoprotein receptor-related protein 5; LRP6, lipoprotein receptor-related protein 6

Key words: propofol, glioma, Wnt signaling, $\beta$-catenin

\section{Introduction}

Wnt signaling is important in embryonic development, tissue homeostasis regulation and tissue regeneration upon injury (1), and the deregulation of Wnt signaling is implicated in the initiation and development of various types of cancer (2). $\beta$-catenin is a key component of Wnt signaling, and its stability and sub-cellular localization are tightly controlled. In the absence of Wnt ligands, cytoplasmic $\beta$-catenin protein is constantly degraded by a destruction complex composed of the scaffolding protein AXIN, adenomatous polyposis coli (APC), casein kinase 1 (CK1) and glycogen synthase kinase $3 \beta$ (GSK3 $\beta$ ) (3). CK1 and GSK3 $\beta$ sequentially phosphorylate $\beta$-catenin, promoting its degradation through an ubiquitination-proteasome pathway (4). The binding of Wnt proteins to frizzled (FZD) receptor and lipoprotein receptor-related protein 5 or 6 (LRP5/6) co-receptors trigger the phosphorylation of LRP5 or LRP6. Phosphorylated LRP5 or LRP6 recruits AXIN to the plasma membrane and inactivates the $\beta$-catenin destruction complex, leading to the accumulation and nuclear translocation of $\beta$-catenin (5). In the nucleus, $\beta$-catenin forms a complex with the lymphoid enhancer factor/T-cell factor and activates the transcription of Wnt target genes, including AXIN2, cyclin D1 (CCND1), B-cell lymphoma 2 (BCL2), naked cuticle homolog 1 (NKD1), and leucine-rich repeat-containing G-protein coupled receptor 5 (LGR5) (6).

Glioma is the most common form of neural malignancy and contributes to $\sim 70 \%$ of malignant primary brain tumors each year (7). Despite therapeutic advances, the prognosis of malignant gliomas remains poor and the majority of patients eventually relapse (8). Increasing evidence demonstrates that the aberrant activation of Wnt signaling is crucial in gliomagenesis $(9,10)$. Hyperactive Wnt signaling in glioma is mainly attributed to the overexpression of positive regulators and the silencing of negative regulators. WNT1, evenness interrupted (EVI), dishevelled 3 , forkhead box M1 (FoxM1) and $\beta$-catenin, primary positive regulators of Wnt signaling, show higher expression in glioma specimens, compared with non-tumor brain tissues, and their expression levels are associated positively with the degree of glioma (11-15). Dickkopf (DKK1 and DKK3), antagonists of Wnt signaling, are frequently silenced through promoter hypermethylation (16). Functional studies have demonstrated that the ectopic expression of positive regulators of Wnt signaling, 
including EVI (12) and FoxM1 (14), promotes glioma tumorigenesis, whereas DKK3 induces cell death in human malignant glioma (17). Therefore, targeting the Wnt signaling may be an effective therapeutic approach to treat patients with glioma.

Propofol is widely used as an intravenous anesthetic agent clinically due to its limited side effects and rapid recovery (18). In addition to its anesthetic advantages, accumulating clinical evidence demonstrates that cancer patients receiving propofol-paravertebral anesthesia during cancer surgery have reduced risk of recurrence and metastasis (19). In addition, studies have shown that propofol exhibits potent antitumor activity in a variety of human cancer cell lines, including lung cancer cells (20), colon carcinoma cells (21), cervical cancer cells (22), pancreatic cancer cells (23) and gastric cancer cells (24), through different molecular mechanisms. However, the effect of propofol on Wnt signaling and glioma cell growth remains to be elucidated. In present study, propofol was identified an inhibitor of Wnt signaling, and it was shown that propofol suppressed the expression of Wnt target genes and growth of glioma cells in vitro and in vivo.

\section{Materials and methods}

Cell culture and reagents. The 293T cells and LN299 glioma cell line were obtained from Cobioer Biosciences Co., Ltd. (Nanjing, China). The LN299 glioma cell line and 293T cells were cultured in Dulbecco's modified Eagle's medium (DMEM; Hyclone; GE Healthcare Life Sciences, Logan, UT, USA) supplemented with $10 \%$ fetal bovine serum (FBS; Hyclone; GE Healthcare Life Sciences), $100 \mathrm{U} / \mathrm{ml}$ penicillin and $100 \mathrm{mg} / 1$ streptomycin. The cells were maintained in humidified incubators at $37^{\circ} \mathrm{C}, 5 \% \mathrm{CO}_{2}$. Propofol was purchased from Sigma-Aldrich; Merck Millipore (Darmstadt, Germany) and dissolved in either DMSO (Sigma-Aldrich; Merck Millipore) or soyabean oil (Santa Cruz Biotechnology, Inc., Santa Cruz, CA, USA).

Luciferase reporter assay. The cells $\left(3 \times 10^{4}\right.$ cells/per well) were plated into 24 -well plates. The following day, the cells were co-transfected with $300 \mathrm{ng}$ of the TOPFlash luciferase construct and $500 \mathrm{ng}$ of control, Wnt1 or Wnt3 plasmid. The pCMX $\beta$ gal plasmid (30 ng) was co-transfected as an internal control. Wnt3a-conditioned medium (Wnt3a-CM) was prepared as previously described (25). At $24 \mathrm{~h}$ post-transfection, the cells were treated the indicated concentrations (1-10 $\mu \mathrm{g} / \mathrm{ml})$ of propofol. Following treatment with propofol for $48 \mathrm{~h}$ in humidified incubators at $37^{\circ} \mathrm{C}$ in $5 \% \mathrm{CO}_{2}$, the cells were harvested for luciferase activity measurement. The luciferase assays were performed using the Dual-Luciferase Reporter Assay system (Promega, Madison, WI, USA) according to the manufacturer's protocol.

Reverse transcription-quantitative polymerase chain reaction (RT-qPCR) analysis. Total RNA was extracted from the 293T and LN299 cells using TRIzol (Qiagen, Inc., Valencia, CA, USA) following the manufacturer's protocol. The RNA (1 $\mu \mathrm{g}$ per sample) was reverse transcribed into cDNA using Reverse Transcriptase M-MLV (Takara Biotechnology Co. Ltd., Dalian, China) according to the manufacturer's protocol. RT-qPCR analysis was performed in an Applied Biosystems
7900 Real-Time PCR system (Applied Biosystems; Thermo Fisher Scientific, Inc., Waltham, MA, USA) using the QuantiTect SYBR Green PCR kit (Qiagen, Inc.) according to the manufacturer's protocols. A total of $0.5 \mu \mathrm{M}$ each primer was added to a $20-\mu 1$ RT-qPCR reaction mix containing $10 \mu \mathrm{l}$ 2x QuantiTect SYBR Green, 500 ng cDNA, 0.5 $\mu$ l QuantiTect RT Mix (Qiagen, Inc.) and RNase-free water. The thermocycling conditions were as follows: $95^{\circ} \mathrm{C}$ for $10 \mathrm{~min}$, followed by 40 cycles of $95^{\circ} \mathrm{C}$ for $30 \mathrm{sec}$ and $60^{\circ} \mathrm{C}$ for $1 \mathrm{~min}$. The average cycle quantitation $(\mathrm{Cq})$, from triplicate assays, was used for further calculations. The endogenous GAPDH used as the normalization control. The $2^{-\Delta \Delta \mathrm{Cq}}$ Method (26) was used to quantify the relative levels of mRNA. The primer sequences were as follows: AXIN2, forward: 5'-CCAACACCAGGCGGA ACGAAG-3' and reverse 5'-CGCCCAATAAGGAGTGTA AGGAC-3'; CCND1, forward 5'-AATGACCCCGCACGA TTTC-3' and reverse 5'-TCAGGTTCAGGCCTTGCAC-3'; NKD1, forward 5'-GGGAAACTTCACTCCAAGCCG' and reverse 5'-GTCTCCCGATCCACTCCTCG-3'; LGR5, forward 5'-CTCTTCCTCAAACCGTCTGC-3' and reverse 5'-GAT CGGAGGCTAAGCAACTG-3'; BCL2, forward 5'-ATGTGT GTGGAGAGCGTCAA-3' and reverse 5'-ACAGTTCCACAA AGGCATCC-3'; GAPDH, forward 5'-CCAGAACATCAT CCCTGCCTCTACT-3' and reverse 5'-GGTTTTTCTAGA CGGCAGGTCAGGT-3'.

Western blot analysis. The cells were pelleted, washed twice in cold PBS and resuspended in $100 \mu$ lysis buffer $(50 \mathrm{mM}$ Tris- $\mathrm{HCl}, \mathrm{pH} 7.4,150 \mathrm{mM} \mathrm{NaCl}, 1 \%$ Nonidet P-40, $1 \mathrm{mM}$ EDTA, $0.25 \%$ sodium deoxycholate and $1 \mathrm{mM} \mathrm{NaF}$ ) supplemented with $1 \mathrm{X}$ protease inhibitors (Roche Diagnostics, Basel, Switzerland) on ice for $30 \mathrm{~min}$. Lysate was centrifuged for $5 \mathrm{~min}$ at $12,000 \times \mathrm{g}, 4^{\circ} \mathrm{C}$ to remove cell debris and the supernatant was harvested. The protein concentration was measured using the Pierce BCA protein assay kit (Thermo Fisher Scientific, Inc.), according to the manufacturer's protocol. The detailed protocol is described previously (27). An equal amount of protein per lane $(50 \mu \mathrm{g})$ were resolved on $10 \%$ SDS-PAGE gels and transferred onto nitrocellulose membranes (Bio-Rad Laboratories, Inc., Richmond, CA, USA). The membranes were blocked with 5\% nonfat milk in PBS containing $0.1 \%$ Tween 20 and then incubated with primary antibodies overnight at $4^{\circ} \mathrm{C}$. Anti-tubulin antibody (cat. no. sc-101527) was purchased from Santa Cruz Biotechnology, Inc.; anti- $\beta$-catenin antibody (cat. no. 9857), anti-phosphorylated (p)LRP6 (Ser1490) antibody (cat. no. 2568), anti-LRP6 antibody (cat. no. 2560), anti-CCND antibody (cat. no. 2922) and anti-BCL2 antibody (cat no. 2872) were obtained from Cell Signaling Technology, Inc. (Beverly, MA, USA). Anti-pLRP6 antibody was used at a dilution of 1:500, anti-tubulin antibody was used at a dilution of 1:200, and other antibodies were used at a dilution of 1:1,000. Following incubation with the horseradish peroxidase-conjugated secondary antibody (cat. no. HAF007 for mouse and cat. no. HAF008 for rabbit; R\&D Systems, Inc., Minneapolis, MN, USA) for $1 \mathrm{~h}$ at room temperature, the proteins were visualized using ECL detection reagent (Amersham; GE Healthcare Life Sciences).

Cell viability assays. The cells were seeded in 96-well plates at a density of 2,000 per well. The following day, the cells were treated with different concentrations $(0,1,2,5$ 

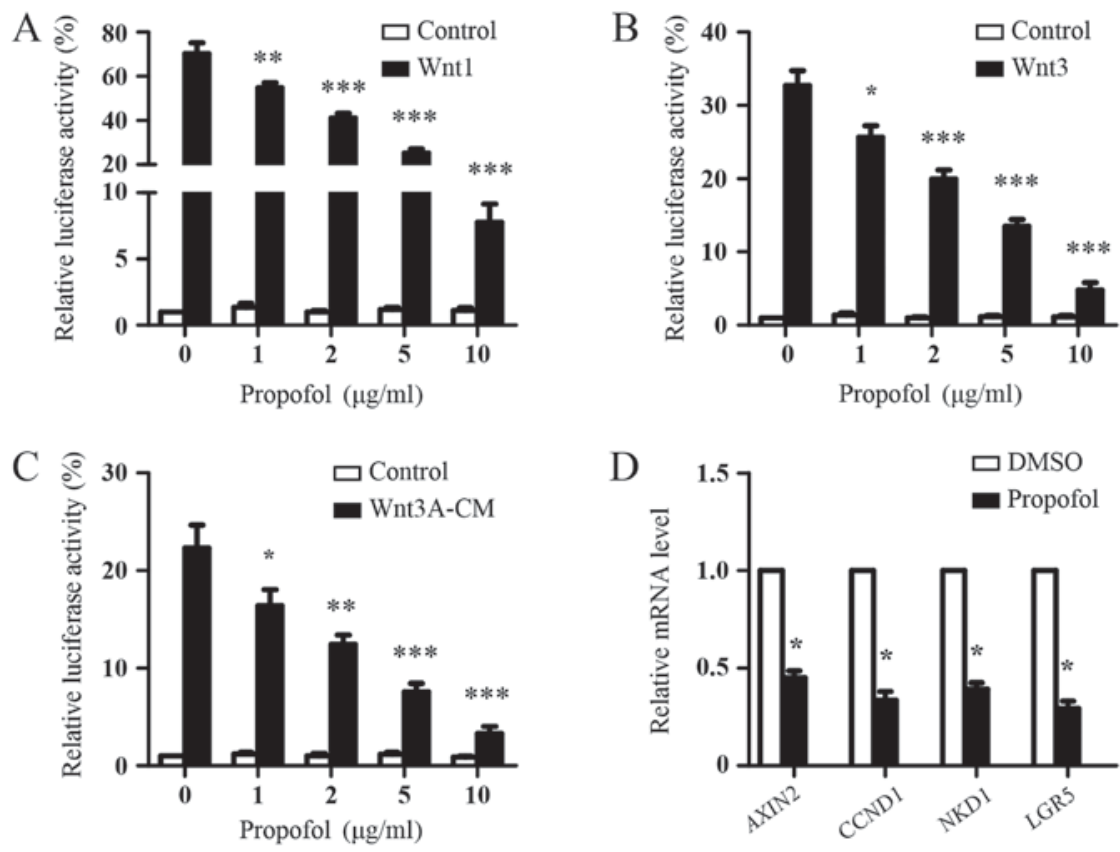

Figure 1. Inhibition of Wnt signaling by propofol. Luciferase reporter assays of 293T cells transfected with the SuperTopFlash reporter plasmid together with (A) Wnt1 or (B) Wnt3 expression plasmids. Following $24 \mathrm{~h}$ of transfection, the cells were treated with indicated concentrations of propofol for $48 \mathrm{~h}$ and luciferase activities were measured. The results are shown as the mean \pm standard deviation of three independent experiments and were analyzed using one-way analysis of variance followed by Dunnett's test. ${ }^{*} \mathrm{P}<0.05,{ }^{* *} \mathrm{P}<0.01$ and ${ }^{* * * *} \mathrm{P}<0.001$, vs. $0 \mu \mathrm{g} / \mathrm{ml}$ propofol. (C) Luciferase reporter assays of $293 \mathrm{~T}$ cells transfected with the SuperTopFlash reporter plasmid. After $24 \mathrm{~h}$, the cells were treated with indicated concentrations of propofol with or without Wnt3A-CM for $48 \mathrm{~h}$ and luciferase activities were measured. Results are shown as the mean \pm standard deviation of three independent experiments. Data were analyzed using one-way analysis of variance followed by Dunnett's test. " $\mathrm{P}<0.05,{ }^{* * *} \mathrm{P}<0.01,{ }^{* * * *} \mathrm{P}<0.001$, vs. $0 \mu \mathrm{g} / \mathrm{ml}$ propofol. (D) RT-qPCR analysis. 293T cells were transfected with Wnt 3 expression plasmids. After $24 \mathrm{~h}$, the cells were treated with DMSO or $5 \mu \mathrm{g} / \mathrm{ml}$ propofol for $48 \mathrm{~h}$, and the mRNA expression of Wnt target genes (AXIN2, CCND1, NKD1 and LGR5) were analyzed using RT-qPCR analysis. DMSO treatment was used as the control. The expression of Wnt target genes was normalized to GAPDH. Data were analyzed using Student's t-test (paired) * $\mathrm{P}<0.05$, vs. DMSO. Wnt3A-CM, Wnt $3 \mathrm{~A}$-conditioned medium; RT-qPCR, reverse transcription-quantitative polymerase chain reaction; CCND1, cyclin D1; NKD1, naked cuticle homolog 1, LGR5, leucine-rich repeat-containing G-protein coupled receptor 5 .

and $10 \mu \mathrm{g} / \mathrm{ml})$ of propofol for $48 \mathrm{~h}$. Cell survival was determined using a Cell Counting Kit-8 (CCK-8) assay (Dojindo Molecular Technologies, Inc., Kumamoto, Japan) according to the manufacturer's protocols. The absorbance at $450 \mathrm{~nm}$ was detected using a multilabel plate reader. Each experiment was performed with three replicates per sample.

Analysis of cell proliferation and apoptosis. Ki67 quantification was performed to determine the effects of propofol treatment on cell proliferation. Briefly, the cells were harvested $48 \mathrm{~h}$ following propofol treatment and stained with anti-human Ki67 antibody (Abcam, Cambridge, MA) at a dilution of 1:200 overnight at $4^{\circ} \mathrm{C}$, followed by a FITC-conjugated mouse anti-rabbit secondary antibody (Thermo Fisher Scientific, Inc.) at a dilution of 1:2,000 for $1 \mathrm{~h}$ at room temperature prior to flow cytometric analysis. Cell apoptosis was assessed using an Annexin V-FITC apoptosis detection kit (BD Pharmingen, San Diego CA, USA) according to the manufacturer's protocols. Briefly, the cells were harvested $48 \mathrm{~h}$ following propofol treatment and washed twice with ice-cold phosphate-buffered saline (PBS). The cells were stained with FITC-labeled Annexin V and PI for $15 \mathrm{~min}$ in the dark, followed by flow cytometric analysis. Data were analyzed using FlowJo software version 10 (Tree Star, Inc., Ashland, OR, USA).

Xenograft animal model. Animal experiments were approved by the Ethics Committee of Yangtze University (Jingzhou,
China). Male, 5-6-week-old immune-deficient BALB/c nude mice were purchased from Vital River Experimental Animal Center (Beijing, China). The mice were housed in specific pathogen-free conditions in an environment with a 12-h light-dark cycle, a temperature of $22 \pm 0.5^{\circ} \mathrm{C}$ and a relative humidity of $50 \pm 1 \%$. The mice had ad libitum access to autoclaved rodent diet and deionized water treated by reverse osmosis. LN299 cells $\left(5 \times 10^{6}\right)$ in $0.1 \mathrm{ml}$ serum-free medium were injected subcutaneously in the right flank of the nude mice. When the tumors reached $\sim 100 \mathrm{~mm}^{3}$, the mice were divided randomly into two groups ( $\mathrm{n}=6 /$ group) according to tumor volumes and body weights, and were treated with the vehicle $(0.5 \mathrm{ml}$ soybean oil) or $0.5 \mathrm{ml}$ propofol $(50 \mathrm{mg} / \mathrm{kg})$ by intraperitoneal injection for 20 days (once each day). The tumor sizes and body weights were measured at indicated times. At the end of the experiments, the mice were sacrificed and tumors were harvested and weighed, followed by RT-qPCR and western blot analyses.

Statistical analysis. All data were analyzed using SPSS 17.0 software (SPSS, Inc., Chicago, IL, USA). All values are presented as the mean \pm standard deviation. Student's t-test (unpaired) was used to detect the statistical significance between two groups. One-way analysis of variance followed by Dunnett's test was used to determine the statistical differences when comparing more than two groups. $\mathrm{P}<0.05$ was considered to indicate a statistically significant difference. 


\section{Results}

Inhibition of Wnt signaling by propofol. The present study first investigated the effect of propofol on the Wnt signaling in 293T cells. Due to the low endogenous Wnt signaling activity in $293 \mathrm{~T}$ cells $(28)$, the $293 \mathrm{~T}$ cells were transfected with the SuperTopFlash reporter plasmid together with Wnt1 or Wnt3 expression plasmids, following which they were treated with increasing concentrations of propofol. As shown in Fig. 1A and $\mathrm{B}$, Wnt1 or Wnt3 transfection significantly increased the transcriptional activity of the SuperTOPflash reporter, compared with the basal levels, and propofol treatment suppressed the Wnt signaling induced by Wnt1 or Wnt3 in a dose-dependent manner. In addition, it was found that propofol dose-dependently inhibited the transcriptional activity of the SuperTopFlash reporter induced with Wnt3A-CM (Fig. 1C). To confirm the inhibitory role of propofol on Wnt signaling, the effect of propofol treatment on Wnt signaling target genes was examined in the 293T cells transfected with Wnt3 expression plasmids using RT-qPCR analysis. Compared with DMSO treatment (as a control), propofol treatment significantly reduced the expression levels of AXIN2, CCND1, NKD1 and LGR5, which are four well-known Wnt targets (Fig. 1D). Taken together, these data suggested that propofol inhibited Wnt signaling.

Propofol suppresses Wnt signaling in glioma cells. The present study then determined the effect of propofol on the Wnt signaling in glioma cells. The LN229 cells were transfected with the SuperTopFlash reporter plasmid and treated with propofol. As shown in Fig. 2A, propofol decreased the transcriptional activity of the SuperTOPflash reporter. In addition, significant decreases in the protein levels of pLRP6, total LRP6 and total $\beta$-catenin were detected in the LN299 cells following propofol treatment, indicating the inhibition of Wnt signaling in the glioma cells by propofol (Fig. 2B). To confirm this inhibitory role, the present study examined the effect of propofol treatment on the expression of Wnt target genes, CCND1 and BLC2, which are two key genes involved in glioma cell proliferation and survival. Using RT-qPCR analysis, it was found that propofol treatment resulted in a marked decrease in the mRNA expression levels of CCND1 and BLC2 in LN299 cells (Fig. 2C). In agreement with the mRNA data, propofol markedly inhibited the protein levels of CCND1 and BCL2 in the glioma cells (Fig. 2D). These results suggested that propofol inhibited Wnt signaling in the glioma cells.

Propofol inhibits proliferation and induces apoptosis in glioma cells. As Wnt signaling is critical in glioma cell viability and it was shown that propofol suppressed Wnt signaling in glioma cells, the present study subsequently investigated the effect of propofol on glioma cell viability using a CCK-8 assay. As shown in Fig. 3A, propofol treatment dose-dependently inhibited the viability of the LN299 cells. Consistent with the inhibitory effect of propofol on the expression of CCND1, propofol treatment resulted in a dose-dependent decrease in the percentage of proliferating LN299 cells, as assessed by Ki67 staining (Fig. 3B). Additionally, propofol treatment led to a significant increase in the percentage of apoptotic LN299
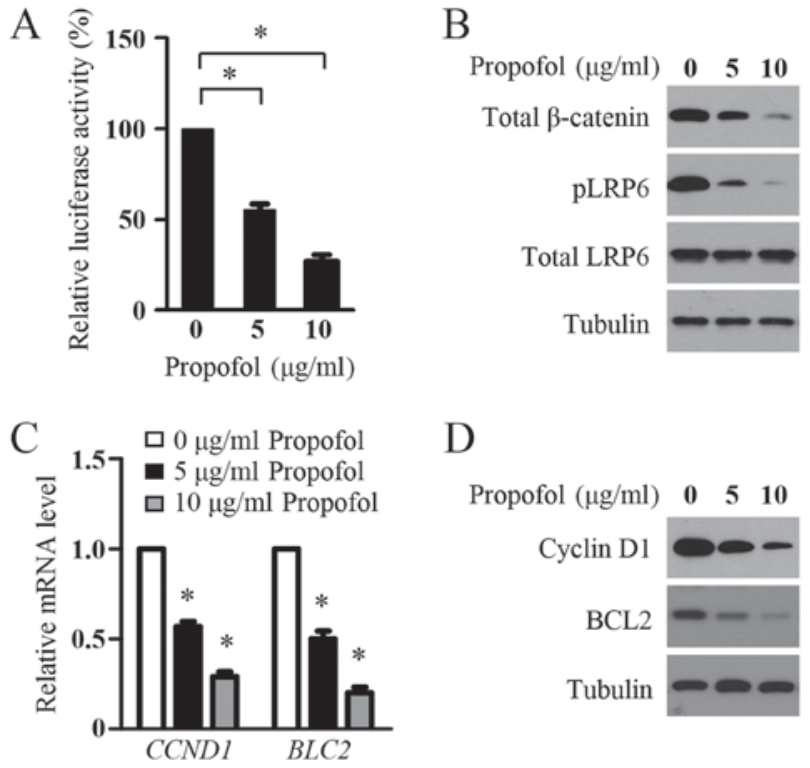

Figure 2. Propofol suppresses Wnt signaling in glioma cells. (A) Luciferase reporter assays. LN299 cells were transfected with the SuperTopFlash reporter plasmid. After 24, the cells were treated with indicated concentrations of propofol for $48 \mathrm{~h}$ and luciferase activities were measured. Results are shown as the mean \pm standard deviation of three independent experiments. ${ }^{*} \mathrm{P}<0.05$. (B) Western blot analysis of protein levels of pLRP6, total LRP6 and total $\beta$-catenin in LN299 cells treated with indicated concentrations of propofol for $48 \mathrm{~h}$. Tubulin was used as a loading control. (C) Reverse transcription-quantitative polymerase chain reaction analysis of mRNA levels of CCND1 and BLC2 in LN299 cells treated with indicated concentrations of propofol for $48 \mathrm{~h}$. The mRNA expression was normalized to GAPDH. Results are shown as the mean \pm standard deviation of three independent experiments. ${ }^{*} \mathrm{P}<0.05$. (D) Western blot analysis of protein levels of CCND1 and BCL2 in LN299 cells treated with indicated concentrations of propofol for $48 \mathrm{~h}$. Tubulin was used as a loading control. LRP6, lipoprotein receptor-related protein 6; pLRP6, phosphorylated LRP6; CCND1, cyclin D1; BCL2, B-cell lymphoma 2.

cells, as measured by Annexin V staining (Fig. 3C). These results indicated that propofol inhibited the proliferation and induced the apoptosis of glioma cells, leading to suppressed glioma cell viability.

Propofol treatment inhibits the growth of xenograft tumors in nude mice and suppresses Wnt signaling in tumors. The above results demonstrated the inhibitory role of propofol on Wnt signaling and cell growth of glioma cells in vitro, therefore, the present study examined whether such an inhibitory effect occurs in vivo. A xenograft tumor model was established by injecting LN299 cells subcutaneously into the right dorsal flank of nude mice. When the average tumor volume reached $\sim 100 \mathrm{~mm}^{3}$, the mice were randomly divided to two groups ( $n=6 /$ group) and treated with an intraperitoneal injection with propofol $(50 \mathrm{mg} / \mathrm{kg})$. Soybean oil was used as a vehicle and to dissolve propofol for treating the xenograft animal, as it is non-toxic and safe for use in mice (29). Propofol treatment significantly suppressed tumor growth (Fig. 4A) but had no effect on body weight (Fig. 4B). Propofol also significantly decreased tumor weight (Fig. 4C). To examine whether propofol treatment suppressed Wnt signaling in the xenograft tumors, the expression levels of Wnt target genes were measured in primary tumors by RT-qPCR analysis. As shown in Fig. 4D, AXIN2, CCND1 
A

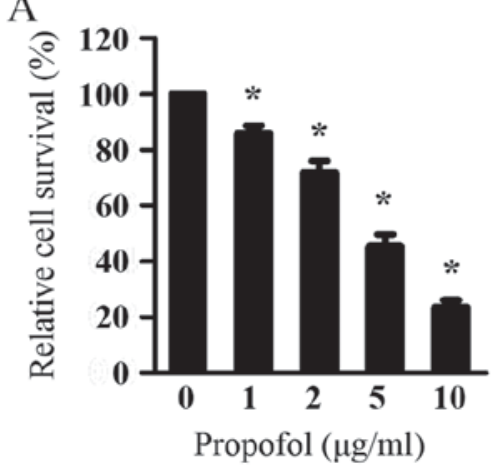

B

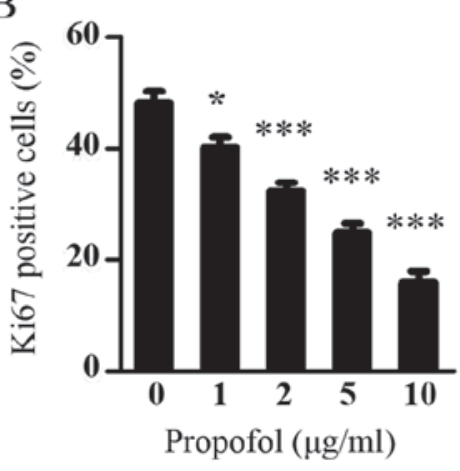

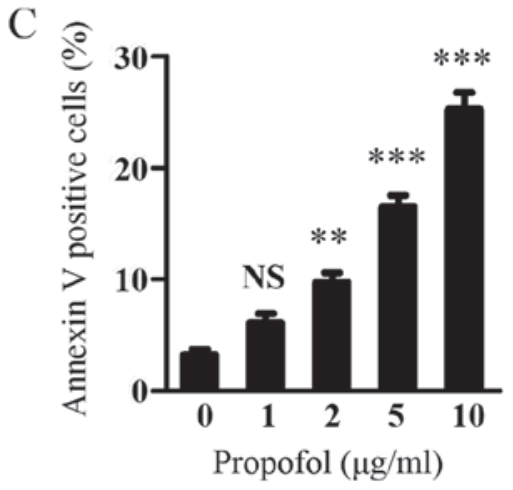

Figure 3. Propofol inhibits proliferation and induces apoptosis in glioma cells. (A) LN299 cells were treated with various concentrations of propofol. Cell Counting Kit- 8 assays were performed following treatment for $48 \mathrm{~h}$. (B) LN299 cells were treated with various concentrations of propofol for $48 \mathrm{~h}$ and cell proliferation was assessed by Ki67 staining. (C) LN299 cells were treated with various concentrations of propofol for $48 \mathrm{~h}$ and cell apoptosis was measured by Annexin V staining. Results are shown as the mean \pm standard deviation of triplicate measurements. Data were analyzed using one-way analysis of variance followed by Dunnett's test. ${ }^{*} \mathrm{P}<0.05,{ }^{* *} \mathrm{P}<0.01,{ }^{* * *} \mathrm{P}<0.001$, vs. $0 \mu \mathrm{g} / \mathrm{ml}$ propofol. NS, not significant.

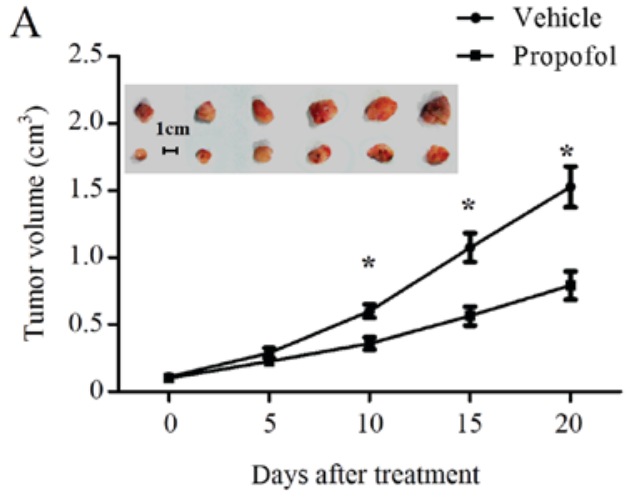

C

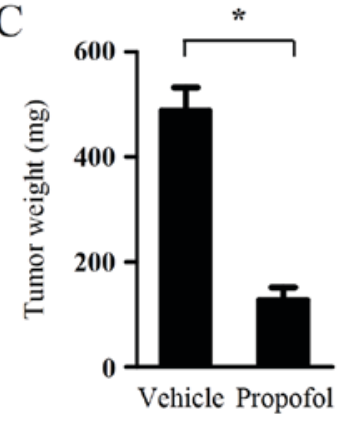

B
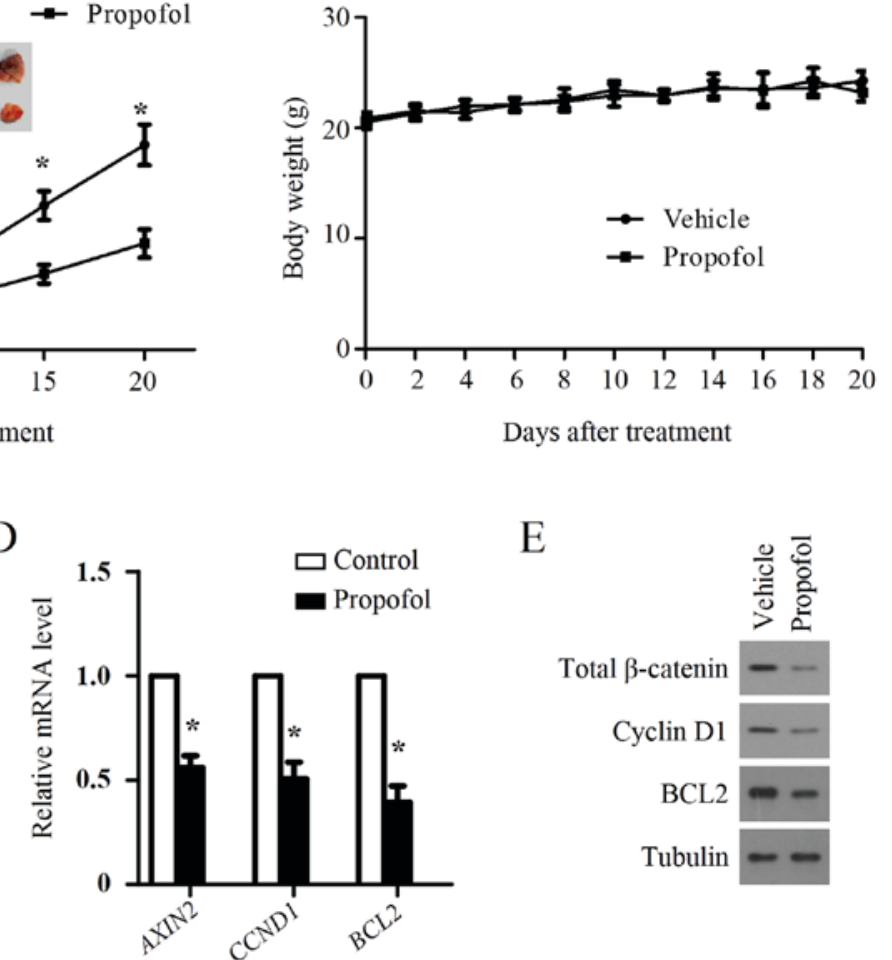

E

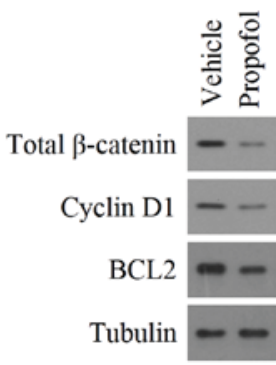

Figure 4. Propofol treatment inhibits the growth of xenograft tumors in nude mice. LN299 cells were injected subcutaneously into the right dorsal flank of nude mice. When the average tumor volume had reached $\sim 100 \mathrm{~mm}^{3}$, the mice were randomly divided to two groups ( $\mathrm{n}=6 / \mathrm{group}$ ) and treated via intraperitoneal injection with propofol $(50 \mathrm{mg} / \mathrm{kg}$ ) or soybean oil (once each day for 20 days). (A) Tumor growth and (B) body weights were measured at the indicated times. ${ }^{*} \mathrm{P}<0.05$. (C) Propofol treatment inhibited LN299 tumor weights. Data are shown as the mean \pm standard deviation (n=6). (D) mRNA expression levels of AXIN2, CCND1 and BCL2 in LN299 xenografts were quantified using reverse transcription-quantitative polymerase chain reaction analysis. The mRNA expression was normalized to GAPDH. (E) Propofol treatment inhibited the protein expression of total $\beta$-catenin, CCND1 and BCL2 in LN299 xenografts. Tubulin was used as a loading control. CCND1, cyclin D1; BCL2, B-cell lymphoma 2.

and BCL2 were significantly downregulated in the LN299 xenografts treated with propofol. In accordance with the mRNA results, propofol treatment also suppressed the protein levels of CCND1 and BCL2 in the LN299 xenografts (Fig. 4E). The protein levels of total $\beta$-catenin in the LN299 xenografts were also repressed by propofol treatment (Fig. 4E), confirming the inhibition of Wnt signaling in LN299 xenografts by propofol treatment.

\section{Discussion}

Propofol is one of the most widely used intravenous anesthetic agents for pain relief in patients in clinical surgery. In addition to its well-known anesthetic advantages, the anticancer effect of propofol has attracted increasing attention (18). In lung cancer cells, propofol can promote cell apoptosis by inducing endoplasmic reticulum stress (20). It has been reported that 
propofol inhibits cervical carcinoma cell growth via decreasing the HOTAIR-mediated mammalian target of rapamycin pathway (22). Propofol has also been found to attenuate prostate cancer cell malignancy through modulating the hypoxia-inducible factor-1 $\alpha$ pathway (30). Li et al demonstrated that propofol reduced the level of matrix metalloproteinase, and suppressed the migration and invasion ability of breast cancer cells via inhibition of the nuclear factor- $\kappa \mathrm{B}$ pathways (31). These findings demonstrate that propofol exerts antitumor activity in several types of cancer through various molecular mechanisms. However, whether propofol has any effects on Wnt signaling and glioma cell growth remains to be elucidated.

Wnt signaling is critical in the initiation and development of glioma. Wnt ligands (Wnt1, Wnt3 and Wnt5) and FZD receptors (FZD2, FZD4, FZD6, FZD7 and FZD9) are overexpressed in human gliomas $(9,10)$. Multiple negative regulators of Wnt signaling, including FAT atypical cadherin 1, dkk1, NKD2, secreted FZD-related protein (sFRP)1 and sFRP2, are downregulated in gliomas (9). Although $\beta$-catenin mutations are rarely detected in gliomas, elevated levels of $\beta$-catenin and high nuclear accumulation are frequently found in glioma tissue and are positively correlated with poor prognosis (32). The transcriptional knockdown of Wnt pathway regulators, including Wnt 2 and $\beta$-catenin, in glioma cells significantly represses cell proliferation and invasiveness, and induces apoptotic cell death (33). SEN461, a potent WNT signaling inhibitor, exhibits anticancer activity in gliomas (34). These findings indicate that WNT signaling is a potential therapeutic target in gliomas.

In the present study, the effect of propofol on WNT signaling was determined using a SuperTopFlash reporter assay and it was found that propofol treatment suppressed the transcriptional activity of the SuperTOPflash reporter in a dose-dependent manner. In addition, propofol significantly inhibited the protein levels of pLRP6 and total $\beta$-catenin and the expression of Wnt target genes in glioma cells, supporting the inhibition of Wnt signaling by propofol. Propofol treatment also inhibited the growth of glioma cells in vitro and in vivo, but had no significant effect on the body weight of propofol-treated mice. Consistent with the inhibitory role of propofol on Wnt signaling in vitro, propofol treatment significantly suppressed Wnt signaling in the xenograft tumors. These data demonstrated that propofol inhibited Wnt signaling and suppressed glioma cell growth. As Wnt signaling is also important in the migration and invasiveness of gliomas, and it was found that propofol decreased Wnt signaling in glioma cells (35), there is merit in examining whether propofol treatment affects glioma cell migration or invasiveness in future investigations.

In conclusion, the data obtained revealed that propofol is a novel potential Wnt signaling inhibitor, which suppressed the growth and survival of glioma cells in vitro and in vivo. The results indicate a novel mechanism of anticancer activity for propofol and provide novel insights into the signaling pathways regulated by propofol. The results suggested the possibility for the potential use of propofol as a novel agent for the treatment of patients with glioma.

\section{Acknowledgements}

The authors would like to thank for Dr. Wei Zhang for his revision of the manuscript.

\section{Funding}

This study was supported by Jingzhou City Science and Technology Funding (grant no. 2016043).

\section{Availability of data and materials}

All data generated or analyzed during this study are included in this published article.

\section{Author's contributions}

RX coordinated and designed the project. WX, JZ, SB, LK, QM, WL, JG and JL performed the experiments. WX and JZ analyzed the data. WX, JZ and RX wrote the manuscript.

\section{Ethics approval and consent to participate}

Animal experiments were approved by the Ethics Committee of Yangtze University (Jingzhou, China).

\section{Consent for publication}

Not applicable.

\section{Competing interests}

The authors declare that they have no competing interests.

\section{References}

1. Zhou Y, Wang Y, Tischfield M, Williams J, Smallwood PM, Rattner A, Taketo MM and Nathans J: Canonical WNT signaling components in vascular development and barrier formation. J Clin Invest 124: 3825-3846, 2014.

2. Anastas JN and Moon RT: WNT signalling pathways as therapeutic targets in cancer. Nat Rev Cancer 13: 11-26, 2013.

3. Chiurillo MA: Role of the Wnt/ $\beta$-catenin pathway in gastric cancer: An in-depth literature review. World J Exp Med 5: 84-102, 2015.

4. Kahn M: Can we safely target the WNT pathway? Nat Rev Drug Discov 13: 513-532, 2014.

5. Zhou L and Liu Y: Wnt/ $\beta$-catenin signalling and podocyte dysfunction in proteinuric kidney disease. Nat Rev Nephrol 11: 535-545, 2015.

6. Inestrosa NC and Varela-Nallar L: Wnt signalling in neuronal differentiation and development. Cell Tissue Res 359: 215-223, 2015.

7. Louis DN: Molecular pathology of malignant gliomas. Ann Rev Pathol 1: 97-117, 2006

8. Omuro A and DeAngelis LM: Glioblastoma and other malignant gliomas: A clinical review. JAMA 310: 1842-1850, 2013.

9. Lee Y, Lee JK, Ahn SH, Lee J and Nam DH: WNT signaling in glioblastoma and therapeutic opportunities. Lab Invest 96: 137-150, 2016.

10. Zhang K, Zhang J, Han L, Pu P and Kang C: Wnt/beta-catenin signaling in glioma. J Neuroimmune Pharmacol 7: 740-749, 2012.

11. Liu C, Tu Y, Sun X, Jiang J, Jin X, Bo X, Li Z, Bian A, Wang X, Liu D, et al: Wnt/beta-Catenin pathway in human glioma: Expression pattern and clinical/prognostic correlations. Clin Exp Med 11: 105-112, 2011.

12. Augustin I, Goidts V, Bongers A, Kerr G, Vollert G, Radlwimmer B, Hartmann C, Herold-Mende C, Reifenberger G, von Deimling A and Boutros M: The Wnt secretion protein Evi/Gpr177 promotes glioma tumourigenesis. EMBO Mol Med 4: 38-51, 2012.

13. Sareddy GR, Panigrahi M, Challa S, Mahadevan A and Babu PP: Activation of Wnt/beta-catenin/Tcf signaling pathway in human astrocytomas. Neurochem Int 55: 307-317, 2009. 
14. Zhang N, Wei P, Gong A, Chiu WT, Lee HT, Colman H, Huang H, Xue J, Liu M, Wang Y, et al: FoxM1 promotes $\beta$-catenin nuclear localization and controls Wnt target-gene expression and glioma tumorigenesis. Cancer Cell 20: 427-442, 2011.

15. Zhang J, Huang K, Shi Z, Zou J, Wang Y, Jia Z, Zhang A, Han L, Yue X, Liu N, et al: High $\beta$-catenin/Tcf-4 activity confers glioma progression via direct regulation of AKT2 gene expression. Neuro Oncol 13: 600-609, 2011.

16. Foltz G, Yoon JG, Lee H, Ma L, Tian Q, Hood L and Madan A: Epigenetic regulation of wnt pathway antagonists in human glioblastoma multiforme. Genes Cancer 1: 81-90, 2010.

17. Mizobuchi Y, Matsuzaki K, Kuwayama K, Kitazato K, Mure H, Kageji T and Nagahiro S: REIC/Dkk-3 induces cell death in human malignant glioma. Neuro Oncol 10: 244-253, 2008.

18. Marik PE: Propofol: Therapeutic indications and side-effects. Curr Pharm Des 10: 3639-3649, 2004.

19. Chen X, Lu P, Chen L, Yang SJ, Shen HY, Yu DD, Zhang XH, Zhong SL, Zhao JH and Tang JH: Perioperative propofol-paravertebral anesthesia decreases the metastasis and progression of breast cancer. Tumour Biol 36: 8259-8266, 2015.

20. Cui WY, Liu Y,Zhu YQ, Song T and Wang QS: Propofol induces endoplasmic reticulum (ER) stress and apoptosis in lung cancer cell H460. Tumour Biol 35: 5213-5217, 2014.

21. Miao Y, Zhang Y, Wan H, Chen L and Wang F: GABA-receptor agonist, propofol inhibits invasion of colon carcinoma cells. Biomed Pharmacother 64: 583-588, 2010.

22. Zhang D, Zhou XH, Zhang J, Zhou YX, Ying J, Wu GQ and Qian JH: Propofol promotes cell apoptosis via inhibiting HOTAIR mediated mTOR pathway in cervical cancer. Biochem Biophys Res Commun 468: 561-567, 2015.

23. Chen X, Wu Q, You L, Chen S, Zhu M and Miao C: Propofol attenuates pancreatic cancer malignant potential via inhibition of NMDA receptor. Eur J Pharmacol 795: 150-159, 2017.

24. Yang C, Gao J, Yan N, Wu B, Ren Y, Li H and Liang J: Propofol inhibits the growth and survival of gastric cancer cells in vitro through the upregulation of ING3. Oncol Rep 37: 587-593, 2017.

25. Willert K, Shibamoto S and Nusse R: Wnt-induced dephosphorylation of axin releases beta-catenin from the axin complex. Genes Dev 13: 1768-1773, 1999.
26. Livak KJ and Schmittgen TD: Analysis of relative gene expression data using real-time quantitative PCR and the 2(-delta delta $\mathrm{C}(\mathrm{T}))$ method. Methods 25: 402-408, 2001.

27. Roberts KP, Ensrud KM and Hamilton DW: A comparative analysis of expression and processing of the rat epididymal fluid and sperm-bound forms of proteins D and E. Biol Reprod 67: 525-533, 2002

28. Wang Z, Li B, Zhou L, Yu S, Su Z, Song J, Sun Q, Sha O, Wang X Jiang $\mathrm{W}$, et al: Prodigiosin inhibits $\mathrm{Wnt} / \beta$-catenin signaling and exerts anticancer activity in breast cancer cells. Proc Nat Acad Sci USA 113: 13150-13155, 2016.

29. Chen H, Khemtong C, Yang X, Chang X and Gao J: Nanonization strategies for poorly water-soluble drugs. Drug Discov Today 16: 354-360, 2011.

30. Huang H, Benzonana LL, Zhao H, Watts HR, Perry NJ, Bevan C, Brown R and Ma D: Prostate cancer cell malignancy via modulation of HIF-1 $\alpha$ pathway with isoflurane and propofol alone and in combination. Br J Cancer 111: 1338-1349, 2014

31. Li Q, Zhang L, Han Y, Jiang Z and Wang Q: Propofol reduces MMPs expression by inhibiting NF- $\kappa \mathrm{B}$ activity in human MDA-MB-231 cells. Biomed Pharmacother 66: 52-56, 2012.

32. Liu X, Wang L, Zhao S, Ji X, Luo Y and Ling F: $\beta$-Catenin overexpression in malignant glioma and its role in proliferation and apoptosis in glioblastma cells. Med Oncol 28: 608-614, 2011.

33. Pu P, Zhang Z, Kang C, Jiang R, Jia Z, Wang G and Jiang H: Downregulation of Wnt2 and beta-catenin by siRNA suppresses malignant glioma cell growth. Cancer Gene Ther 16: 351-361, 2009.

34. Sareddy GR, Kesanakurti D, Kirti PB and Babu PP: Nonsteroidal anti-inflammatory drugs diclofenac and celecoxib attenuates Wnt $/ \beta$-catenin/Tcf signaling pathway in human glioblastoma cells. Neurochem Res 38: 2313-2322, 2013.

35. Paul I, Bhattacharya S, Chatterjee A and Ghosh MK: Current understanding on EGFR and Wnt/ $\beta$-Catenin signaling in glioma and their possible crosstalk. Genes Cancer 4: 427-446, 2013. 